\title{
Long non-coding RNA SNHG20 promotes nasopharyngeal carcinoma cell migration and invasion by upregulating TGF-ß1
}

\author{
CAIBO SUN, YUNING SUN and ENDONG ZHANG \\ Department of Otolaryngology, The Second Municipal Hospital of Wei Hai Affiliated to Qing Dao University, \\ Weihai, Shandong 264200, P.R. China
}

Received April 24,2018; Accepted August 2, 2018

DOI: $10.3892 /$ etm.2018.6849

\begin{abstract}
Small nucleolar RNA host gene 20 (SNHG20) has been reported to serve roles in several types of malignancies, while its role in nasopharyngeal carcinoma remains unknown. In the present study, tumor tissues and adjacent healthy tissues of patient with nasopharyngeal carcinoma, as well as blood samples from patients with nasopharyngeal carcinoma and heathy controls were collected, and expression levels of SNHG20 were detected by reverse transcription-quantitative polymerase chain reaction. Receiver operating characteristic curve and survival curve analyses were performed to evaluate the diagnostic and prognostic values of SNHG20 expression for nasopharyngeal carcinoma, respectively. Associations between serum expression levels of SNHG20 and clinical data of patients with nasopharyngeal carcinoma were analyzed using $\chi^{2}$ test. A SNHG20 expression vector was constructed and transfected into nasopharyngeal carcinoma cells, and cell migration and invasion were detected by Transwell assays. Expression of transforming growth factor- $\beta 1$ (TGF- $\beta 1$ ) was detected by western blotting. Results indicated that the expression level of SNHG20 increased in cancer tissues compared with healthy tissues of patients with nasopharyngeal carcinoma. Serum level of SNHG20 increased in patients with nasopharyngeal carcinoma compared with healthy controls. Significant association was identified between serum levels of SNHG20 and distant tumor metastasis. Serum SNHG20 could serve as a potential diagnostic and prognostic marker for nasopharyngeal carcinoma. Overexpression of SNHG20 promoted nasopharyngeal carcinoma cell migration and invasion, and promoted the expression of TGF- $\beta 1$. TGF- $\beta 1$ inhibitor reduced the effects of SNHG20 overexpression on nasopharyngeal carcinoma cell migration and invasion, and exhibited no significant effect on SNHG20 expression. Therefore,
\end{abstract}

Correspondence to: Dr Endong Zhang, Department of Otolaryngology, The Second Municipal Hospital of Wei Hai Affiliated to Qing Dao University, 42 Wenhua East Road, Weihai, Shandong 264200, P.R. China

E-mail: jirzeg3@163.com

Key words: nasopharyngeal carcinoma, long noncoding RNA small nucleolar RNA host gene 20 , transforming growth factor- $\beta 1$, migration, invasion the results of the present study indicated that lncRNA SNHG20 could promote the migration and invasion of nasopharyngeal carcinoma cells by upregulating TGF- $\beta 1$.

\section{Introduction}

Nasopharyngeal carcinoma is the most common malignancy of the nasopharynx (1). Although nasopharyngeal carcinoma is a rare malignancy with an incidence rate below $1 / 100,000$ in most regions of the world, it is considered to be a major cancer in some areas (2). Particularly, China has the highest incidence of nasopharyngeal carcinoma globally, and the age at onset of nasopharyngeal carcinoma in China is decreasing (3). As a result of efforts to treat nasopharyngeal carcinoma, survival of patients with this disease has improved markedly during the last several decades (4). However, more than $30 \%$ of patients with nasopharyngeal carcinoma in Southern China develop tumor metastasis and recurrence even after radical treatment (5), leading to poor survival rates of those patients. Therefore, early diagnosis and accurate prognosis remain essential for the survival of patients with nasopharyngeal carcinoma.

The development of nasopharyngeal carcinoma is a complex multistep process with various internal and external factors involved (6). It has been reported that the onset, development and progression of nasopharyngeal carcinoma is associated with alterations in the expression levels of certain long non-coding RNAs (lncRNAs) (7). LncRNAs are a subgroup of non-coding RNAs composed of more than 200 nucleotides which have been recognized to serve roles in both normal physical processes and pathological alterations (8). Small nucleolar RNA host gene 20 (SNHG20) is a recently identified lncRNA with an oncogenic role in hepatocellular carcinoma (9) and colorectal cancer (10). In the present study, IncRNA SNHG20 promoted the migration and invasion of nasopharyngeal carcinoma cells by upregulating transforming growth factor- $\beta 1$ (TGF- $\beta 1)$.

\section{Patients and methods}

Patients. The present study included 55 patients with nasopharyngeal carcinoma who were pathologically diagnosed and treated for the first time in the Wei Hai Hospital affiliated to the Medical College of Qing Dao University (Weihai, China) 
form January 2011 to February 2013. The patients included 30 males and 25 females. Distant tumor metastasis was observed in 28 cases. The age of patients ranged from 26 to 72 years with an average age of $50.4 \pm 11.1$ years. Patients with other types of malignancies and other severe diseases were excluded. Furthermore, 40 healthy volunteers were included to serve as the control group. The control group included 22 males and 18 females (age range, 25-69 years; average age, $51.1 \pm 10.7$ years). No significant differences in age and sex distributions were identified between the two groups. Before the study, all participants were informed of the experimental protocols and each patient signed informed consent. The present study was approved by the Ethics Committee of Wei Hai Hospital affiliated to the Medical College of Qing Dao University.

Specimen collection. Tumor tissues and adjacent healthy tissues within $2 \mathrm{~cm}$ around the tumors were colletcted from patients with nasopharyngeal carcinoma during surgical resection. Blood $(10 \mathrm{ml})$ was extracted from the elbow vein of patients with nasopharyngeal carcinoma and healthy controls on the day of admission. Following incubation at room temperature for $2 \mathrm{~h}$, blood samples were centrifuged at room temperature for $20 \mathrm{~min}$ at $1,000 \mathrm{x}$ g to collect serum. All specimens were stored in liquid nitrogen for further use.

Cell lines and cell culture. Two nasopharyngeal carcinoma cell lines 13-9B and C666-1 NP69 were purchased from American Type Culture Collection (Manassas, VA, USA). A normal nasopharyngeal cell line NP69 was purchased from Xiang Ya Central Experiment Laboratory (Changsha, China; http://gdyjzx.csu.edu.cn/zxjs/sysjs/xbs.htm). Cells were cultured in RPMI-1640 medium (cat. no. 30-2001; American Type Culture Collection) contaning $10 \%$ fetal bovine serum (Thermo Fisher Scientific, Inc., Waltham, MA, USA) at $37^{\circ} \mathrm{C}$ and $5 \% \mathrm{CO}_{2}$. Serum was not added in case of drug treatment. Cells were also treated with a TGF- $\beta$ inhibitor, LY2109761 (100 nM; Sigma-Aldrich; Merck KGaA, Darmstadt, Germany), for $24 \mathrm{~h}$ at $37^{\circ} \mathrm{C}$ and $5 \% \mathrm{CO}_{2}$.

Reverse transcription-quantitative polymerase chain reaction $(R T-q P C R)$. Total RNA was extracted from tumor tissues, adjacent healthy tissues and serum using TRIzol reagent (Invitrogen; Thermo Fisher Scientific, Inc.). Tumor tissues and adjacent healthy tissues were ground in liquid nitrogen prior to the addition of the TRIzol reagent. NanoDrop $^{\text {TM }} 2000$ Spectrophotometer (Thermo Fisher Scientific, Inc.) was used to test all RNA samples and samples with a A260/A280 ratio of 1.8-2.0 were subjected to RT using Superscript IV Reverse Transcriptase (Thermo Fisher Scientific, Inc.) to synthesize cDNA. PCR reaction system was prepared using SYBR ${ }^{\mathrm{TM}}$ Green Real-Time PCR Master Mix (Thermo Fisher Scientific, Inc.). Sequences of primers used for PCR reactions were: IncRNA SNHG20 forward, 5'-ATGGCTATAAATAGATACACGC-3' and reverse, 5'-GGTACAAACAGGGAGGGA-3'; matrix metalloproteinase (MMP)-2 forward, 5'-CTCAGATCCGTGGTG AGATCT-3' and reverse, 5'-CTTTGGTTCTCCAGCTTC AGG-3"; MMP-9 forward, 5'-ATCCAGTTTGGTGTCGCG GAGC-3" and reverse, 5'-GAAGGGGAAGACGCACAG
CT-3"; $\beta$-actin forward, 5'-GACCTCTATGCCAACACA GT-3" and reverse, 5'-AGTACTTGCGCTCAGGAGGA-3". $\mathrm{PCR}$ reaction conditions were: Initial denaturation at $95^{\circ} \mathrm{C}$ for $40 \mathrm{sec} ; 40$ cycles of $95^{\circ} \mathrm{C}$ for $12 \mathrm{sec}$ and $58^{\circ} \mathrm{C}$ for $42 \mathrm{sec}$. Relative expression levels of 1ncRNA SNHG20, MMP-2 and MMP-9 were normalized to endogenous control $\beta$-actin using the $2^{-\Delta \Delta \mathrm{Cq}}$ method (11).

Construction of the SNHG20 expression vector and transfection. An EcoRI-EcoRI fragment containing SNHG20 cDNA amplified by PCR was inserted into a pIRSE2-EGFP vector (Clontech Laboratories, Inc., Mountainview, CA, USA) to construct a SNHG20 expression vector. Cells were harvested during the logarithmic growth phase and cultured overnight to reach $80-90 \%$ confluence. Lipofectamine ${ }^{\circledR} 2000$ reagent (cat. no. 11668-019; Invitrogen; Thermo Fisher Scientific, Inc.) was used to transfect $10 \mathrm{mM}$ vector into $5 \times 10^{5}$ cells. Cells were cultured for $48 \mathrm{~h}$ prior to subsequent experiments. Untransfected cells were used as the control group. Cells transfected with empty vectors were the negative control group. The overexpression rate of SNHG20 was $>200 \%$ compared with untransfected cells, which was determined prior to the current study (data not shown).

Cell migration and invasion assays. Cell migration ability was measured using a Transwell cell migration assay kit (BD Biosciences, Franklin Lakes, NJ, USA). Cells were harvest during the logarithmic growth phase and single cell suspension was prepared with a cell density of $5 \times 10^{4}$ cells per $1 \mathrm{ml} \mathrm{RPMI-1640}$ medium. Subsequently, $0.1 \mathrm{ml}$ cell suspension containing $5 \times 10^{3}$ cells was added into the upper chamber, RPMI-1640 (Thermo Fisher Scientific, Inc.) supplemented with $20 \%$ fetal calf serum (Sigma-Aldrich; Merck KGaA) was used to fill the lower chamber. After incubation for $24 \mathrm{~h}$, membranes were collected and subjected to staining with $0.5 \%$ crystal violet (Sigma-Aldrich; Merck KGaA) for $20 \mathrm{~min}$ at room temperature. Membranes were observed under an optical microscope (Olympus Corporation, Tokyo, Japan) and the stained cells were counted. The upper chamber was pre-coated with Matrigel (cat. no. 356234; Millipore, USA) before the cell invasion assay, all other steps were the same as for the migration assay. Cell migration and invasion were normalized to the group with the largest number of migrating and invading cells, which was set to 100 .

Western blot analysis. Cells were harvest during the logarithmic growth phase and total protein was extracted using RIPA solution (Thermo Fisher Scientific, Inc.). Bicinchoninic acid assay was performed for protein determination. Subsequently, $10 \%$ SDS-PAGE was performed with $20 \mu \mathrm{g}$ protein per lane. After the gel transfer, polyvinylidene fluoride membranes were blocked with $5 \%$ skimmed milk at room temperature for $1 \mathrm{~h}$. Following blocking, membranes were incubated with rabbit anti-TGF- $\beta 1$ (cat. no. ab92486), $\beta$-catenin (cat. no. ab16051), Akt (cat. no. ab126811), pan-AKT (phospho T308; cat. no. ab38449), phospho anti-NF-кB p65 (S536; cat. no. ab28856; all 1:1,500), and GAPDH (1:1,000; cat. no. ab9485; all Abcam, Cambridge, UK) antibodies overnight at $4^{\circ} \mathrm{C}$. The following day, the membranes were incubated with goat anti-rabbit immunoglobulin G-horseradish 


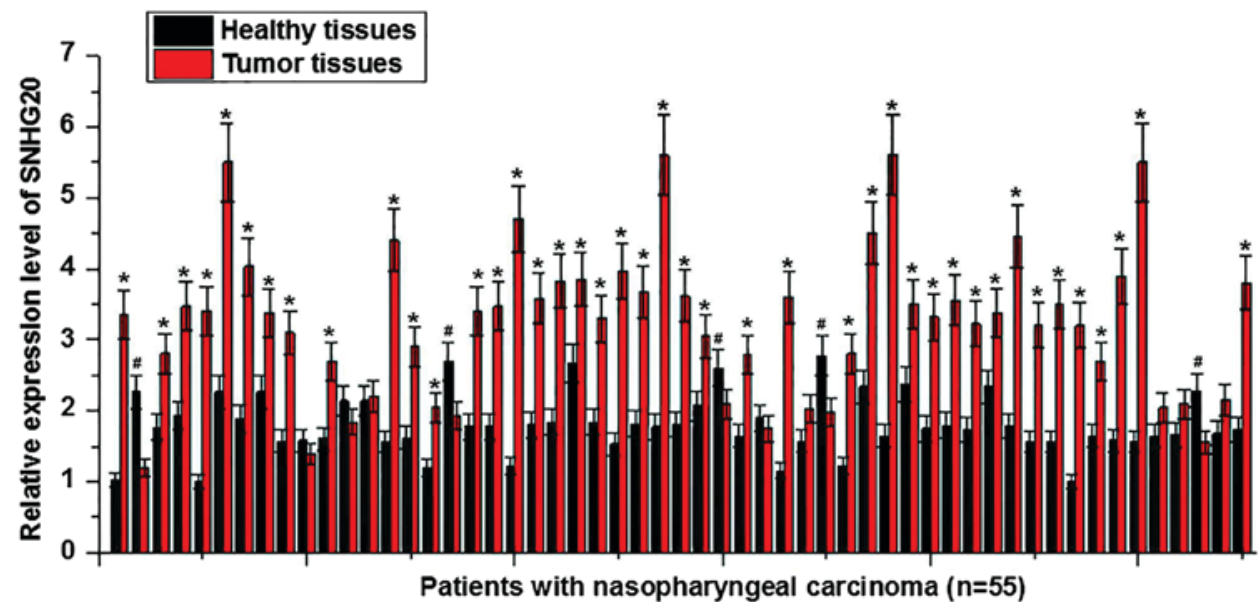

Figure 1. Expression of long noncoding RNA SNHG20 in tumor tissues and adjacent healthy tissues of 55 patients with nasopharyngeal carcinoma. ${ }^{*} \mathrm{P}<0.05$ vs. the adjacent healthy tissues and ${ }^{\#} \mathrm{P}<0.05$ vs. tumor tissues. SNHG20, small nucleolar RNA host gene 20.
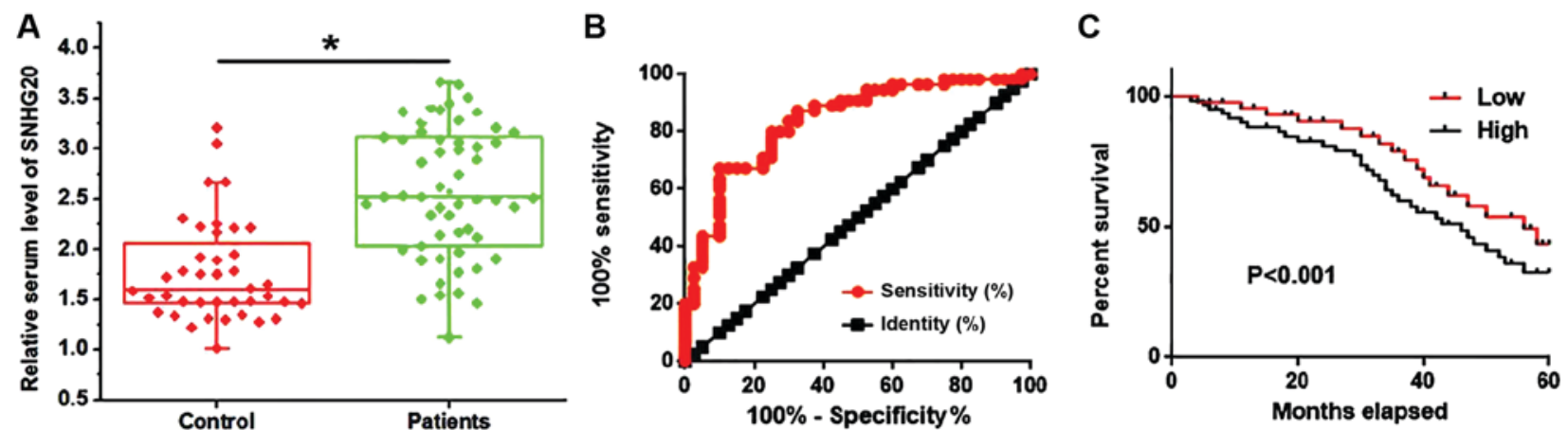

Figure 2. Serum level of SNHG20 in patients with nasopharyngeal carcinoma and healthy controls and the diagnostic and prognostic values of serum SNHG20 for nasopharyngeal carcinoma. (A) Serum levels of SNHG20 in patients with nasopharyngeal carcinoma and healthy controls. (B) Receiver operating characteristic curve of the use of serum SNHG20 in the diagnosis of nasopharyngeal carcinoma. (C) Survival curve of patients with high and low serum expression levels of SNHG20. "P<0.05. SNHG20, small nucleolar RNA host gene 20.

peroxidase-conjugated secondary antibody (1:1,000; cat. no. MBS435036; MyBioSource, Inc., San Diego, CA, USA) at room temperature for $2 \mathrm{~h}$. Enhanced chemiluminescence reagent (Sigma-Aldrich; Merck KGaA) was added to develop the signal. Relative expression level of TGF- $\beta 1$ was normalized to the endogenous GAPDH control using ImageJ (version 1.6; National Institutes of Health, Bethesda, MD, USA).

Statistical analysis. SPSS software (version 19.0; IBM Corp., Armonk, NY, USA) was used for data analysis. All experiments were performed in triplicate manner. Data are presented as the mean \pm standard deviation. Comparisons between two groups were performed using a t-test and comparisons among multiple groups were performed using one-way analysis of variance, followed by Least Significant Difference post-hoc test. Count data were compared using $\chi^{2}$ test In $\chi^{2}$ test, patients with smoking habits were defined as $>5$ cigarettes per day, 4 times per week. Patients with drinking habits were defined as drinking $>2$ times per week. Patients were divided into the high $(n=28)$ and low $(n=27)$ expression groups according to the median serum level of SNHG20 and the Kaplan-Meier method was used to draw the survival curves. Survival curves were compared using log-rank test. $\mathrm{P}<0.05$ was considered to indicate a statistically significant difference.

\section{Results}

Expression of IncRNA SNHG20 in tumor tissue and adjacent healthy tissues of 55 patients with nasopharyngeal carcinoma. Expression levels of SNHG20 in tumor tissues and adjacent healthy tissues of 55 patients with nasopharyngeal carcinoma were detected by RT-qPCR. The expression level of SNHG20 was found to be significantly higher in tumor tissues compared with adjacent healthy tissues $(\mathrm{P}<0.05)$ in 42 out of 55 patients (Fig. 1). The expression level of SNHG20 was significantly lower in tumor tissues compared with adjacent healthy tissues $(\mathrm{P}<0.05)$ of 5 patients. No significant differences in the expression levels of SNHG20 between tumor tissues and adjacent healthy samples were found in the remaining 8 patients. These results suggested that upregulation of SNHG20 may be involved in the pathogenesis of nasopharyngeal carcinoma.

Serum expression level of SNHG2O and the diagnostic and prognostic values of serum SNHG20 for nasopharyngeal carcinoma. Serum levels of SNHG20 in patients with nasopharyngeal carcinoma and healthy controls were measured by RT-qPCR. Serum expression levels of SNHG20 were significantly higher in patients with nasopharyngeal carcinoma compared with healthy controls $(\mathrm{P}<0.05)$ (Fig. 2A). Receiver operating characteristic 
Table I. Associations between serum levels of small nucleolar RNA host gene 20 and clinicopathological characteristics of patients.

\begin{tabular}{lcccccc}
\hline Characteristic & Group & Cases, $\mathrm{n}$ & High-expression & Low-expression & $\chi^{2}$ & P-value \\
\hline Sex & Male & 30 & 18 & 12 & 2.18 & 0.14 \\
\multirow{2}{*}{ Age, years } & Female & 25 & 10 & 15 & & \\
& $>50$ & 29 & 13 & 16 & 0.91 & 0.34 \\
Distant tumor metastasis & $<50$ & 26 & 15 & 11 & & \\
\multirow{5}{*}{ Smoking } & Yes & 28 & 19 & 9 & 6.56 & 0.01 \\
\multirow{4}{*}{ Alcohol consumption } & No & 27 & 9 & 18 & & \\
& Yes & 32 & 15 & 17 & 0.49 & 0.48 \\
& No & 23 & 13 & 17 & 0.85 & 0.36 \\
\hline
\end{tabular}

curve analysis indicated that the area under the curve of the use of serum SNHG20 in the diagnosis of nasopharyngeal carcinoma was 0.8377 with $95 \%$ confidence interval of $0.7563-0.9191$ $(\mathrm{P}<0.0001)$ (Fig. 2B). The 55 patients were followed-up for 60 months to record their survival. Patients were divided into the high $(n=28)$ and low $(n=27)$ expression groups according to the median serum level of SNHG20. Kaplan-Meier method was used to plot the survival curves for both groups, followed by comparison of survival curves between the two groups by log rank test. The overall survival rate of patients with the high expression level of SNHG20 was significantly lower compared with patients with the low expression level $(\mathrm{P}<0.001)$ (Fig. 2C). These results suggested that serum expression level of SNHG20 may serve as a diagnostic and prognostic marker for nasopharyngeal carcinoma.

Association between serum expression levels of SNHG2O and clinicopathological data of patients. Patients were divided into the high $(n=28)$ and low $(n=27)$ expression groups according to the median serum level of SNHG20. Associations between serum expression levels of 1ncRNA SNHG20 and clinicopathological data of patients were analyzed by $\chi^{2}$ test. Expression levels of lncRNA SNHG20 in serum exhibited no significant association with patients' sex, age, alcohol consumption and smoking habits, and were significantly associated with distant tumor metastasis $(\mathrm{P}<0.05$; Table I).

Possible interaction between SNHG20 and TGF- $\beta 1$ in nasopharyngeal carcinoma. The TGF- $\beta$ pathway serves roles in tumor metastasis of various types of cancer (12). The aforementioned data suggested that upregulated expression of SNHG20 may be involved in the metastasis of nasopharyngeal carcinoma. Therefore, in the present study, a SNHG20 expression vector was transfected into nasopharyngeal carcinoma cell lines 13-9B and C666-1, and a normal human nasopharyngeal epithelial cell line NP69, and the possible interactions between TGF- $\beta 1$ and SNHG20 were studied. Overexpression of SNHG20 significantly increased the expression level of active TGF- $\beta 1$ in nasopharyngeal carcinoma cell lines 13-9B and C666-1 $(\mathrm{P}<0.05)$, and not in the normal human nasopharyngeal epithelial cell line NP69 (Fig. 3A). By contrast, treatment with TGF- $\beta 1(10 \mathrm{ng} / \mathrm{ml})$ induced no significant effect on SNHG20 in all cell lines included in the present study (Fig. 3B). These results suggested that SNHG20 may serve as an upstream activator of TGF- $\beta 1$ in nasopharyngeal carcinoma. In addition, in the present study, oncogenic signaling pathways including Wnt, Akt and $\mathrm{NF}-\kappa \mathrm{B}$ failed to respond following SNHG20 overexpression (data not shown).

Effects of SNHG20 overexpression on the expression of markers of epithelial-mesenchymal transition (EMT). RT-qPCR was performed to detect the expression of markers of EMT including MMP-2 and MMP-9 following SNHG20 overexpression. SNHG20 overexpression significantly promoted the expression of MMP-2 (Fig. 4A) and MMP-9 (Fig. 4B) in nasopharyngeal carcinoma cell lines 13-9B and C666-1, and not cells of the normal human nasopharyngeal epithelial cell line NP69.

Effects of SNHG2O overexpression and treatment with TGF- $\beta$ inhibitor on cell migration and invasion. Transwell migration and invasion assays were carried out to investigate the effects of SNHG20 overexpression on migration and invasion of nasopharyngeal carcinoma cell lines 13-9B and C666-1 and a normal human nasopharyngeal epithelial cell line NP69. SNHG20 overexpression significantly promoted migration (Fig. 5A) and invasion (Fig. 5B) of nasopharyngeal carcinoma cell lines 13-9B and C666-1 compared with the $\mathrm{C}$ and NC groups. SNHG20 overexpression did not promote migration and invasion in the normal human nasopharyngeal epithelial cell line NP69. However, compared with cells with SNHG20 overexpression alone, nasopharyngeal carcinoma cells overexpressing SNHG20 treated with TGF- $\beta$ inhibitor LY2109761 (100 nM; Sigma-Aldrich; Merck KGaA) exhibited significantly reduced migrtaion (Fig. 5A) and invasion (Fig. 5B) rates. Therefore, the TGF- $\beta$ inhibitor LY2109761 significantly reduced the effects of SNHG20 overexpression on migration and invasion of nasopharyngeal carcinoma cells. 

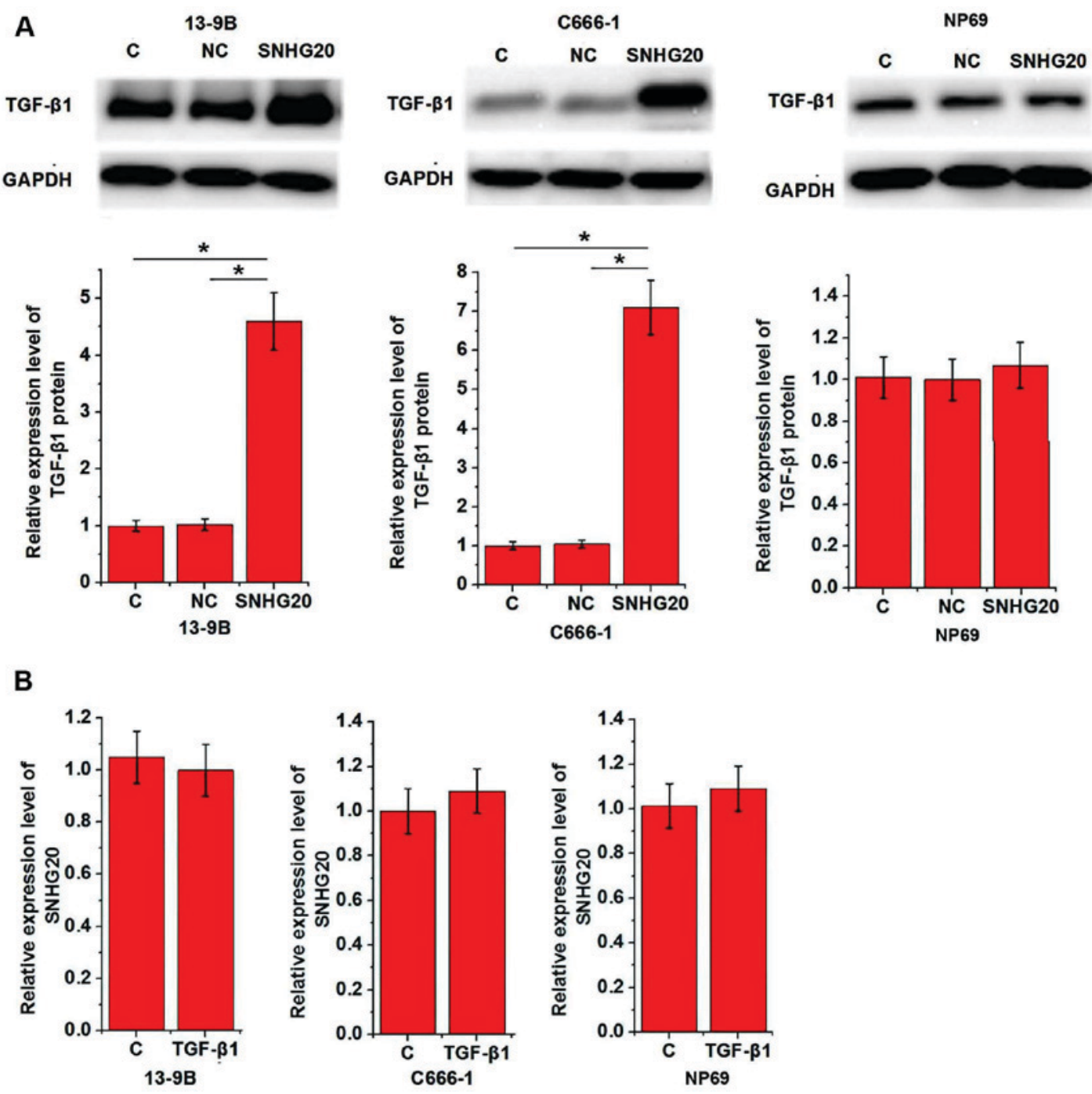

Figure 3. Possible interaction between SNHG20 and TGF- $\beta 1$ in nasopharyngeal carcinoma. (A) The effects of SNHG20 overexpression on the expression levels of active TGF- $\beta 1$ protein. (B) The effect of treatment with TGF- $\beta 1$ on the expression levels of SNHG20 in different cell lines. "P<0.05. SNHG20, small nucleolar RNA host gene 20; C, untransfected cells; NC, cells transfected with empty vectors; TGF- $\beta 1$, transforming growth factor- $\beta 1$.

\section{Discussion}

The present study revealed that lncRNA SNHG20 may be involved in the pathogenesis of nasopharyngeal carcinoma, which is a common malignancy in China (2). The present study also suggested that detection of SNHG20 expression may be performed to improve the diagnosis and prediction of prognosis of patients with nasopharyngeal carcinoma.

The expression levels of SNHG20 were detected in nasopharyngeal carcinoma tissues and adjacent healthy tissues of patients included in the present study. It has been previously reported that SNHG20 was upregulated in hepatocellular carcinoma tissues compared with non-tumor liver tissues (9). Upregulated expression of SNHG20 was also observed in colorectal cancer tissues (10). Those two previous studies indicated that SNHG20 may be an oncogenic IncRNA.In the present study, a significantly increased expression level of SNHG20 was observed in the majority of tumor tissues compared with the adjacent healthy tissues (42 out of 55 patients accounting for $76.4 \%$ ). The expression level of SNHG20 was significantly lower in the tumor tissues compared with the adjacent healthy tissues in 5 patients, only accounting for $9 \%$. These results suggested that upregulation of 1ncRNA SNHG20 may be involved in the pathogenesis of nasopharyngeal carcinoma.

Tumor metastasis and postoperative recurrence severely affect patients' survival (5). Therefore, early diagnosis and treatment are essential. Development of human diseases is usually accompanied by alterations of expression of certain substances in the blood, and detecting the levels of those substances may provide guidance for the diagnosis and prognosis of diseases $(13,14)$. In the present study, significantly increased serum expression levels of IncRNA SNHG20 were observed in patients with nasopharyngeal carcinoma compared with healthy controls. Furthermore, patients with the high serum expression level of SNHG20 exhibited significantly shortened survival time compared with the low serum expression group. These results suggested that serum lncRNA SNHG20 may serve as a potential diagnostic and prognostic biomarker for nasopharyngeal carcinoma. However, it has been previously demonstrated that expression of lncRNAs was affected by aging (15), tobacco consumption (16) and alcohol abuse (17). Therefore, age and living habits of patients may affect the accuracy of serum expression of lncRNA 

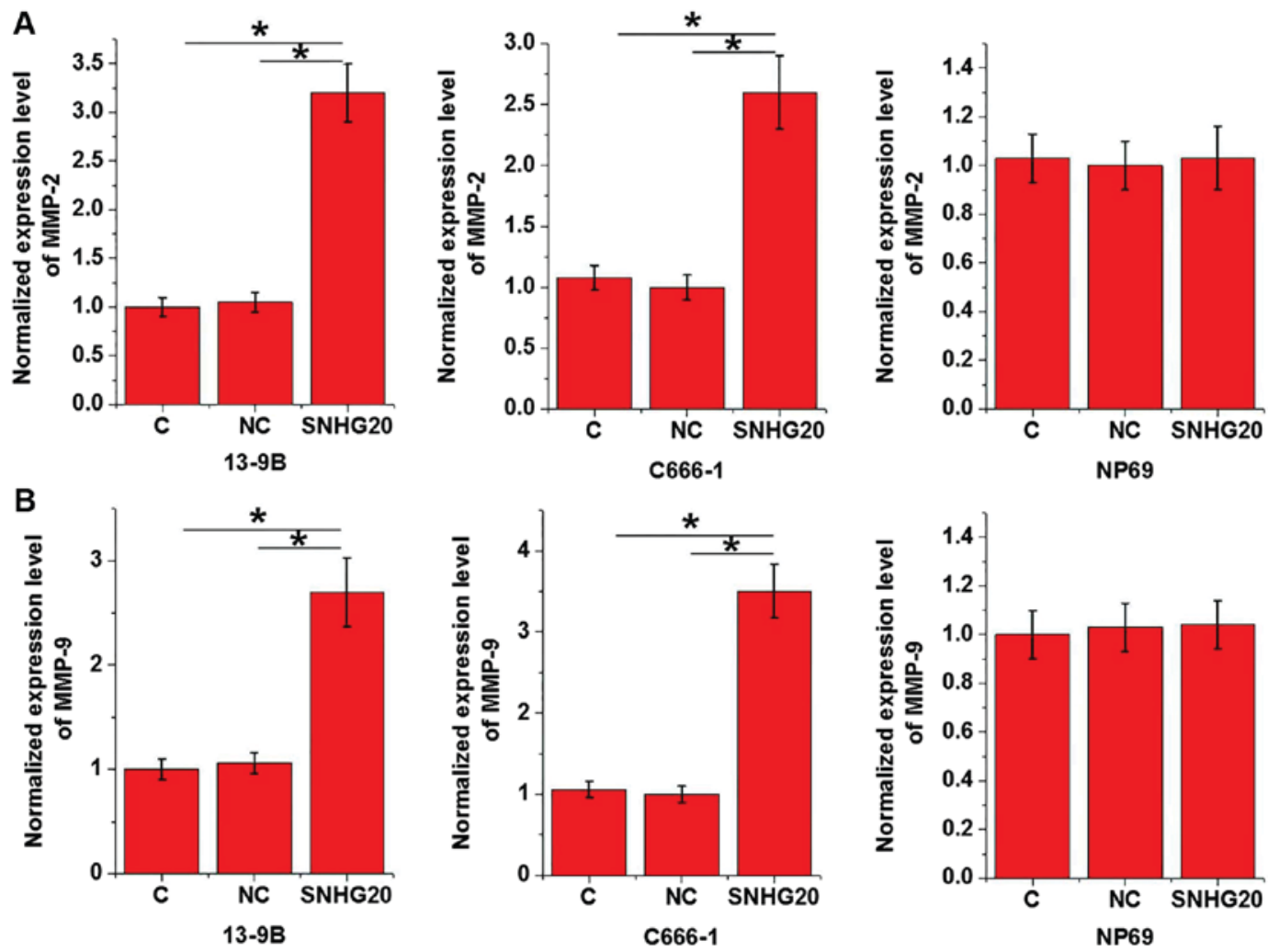

Figure 4. Effects of SNHG20 overexpression on markers of epithelial-mesenchymal transition. Expression levels of (A) MMP-2 and (B) MMP-9. * $<0.05$. SNHG20, small nucleolar RNA host gene 20; C, untransfected cells; NC, cells transfected with empty vectors; MMP, matrix metalloproteinase.
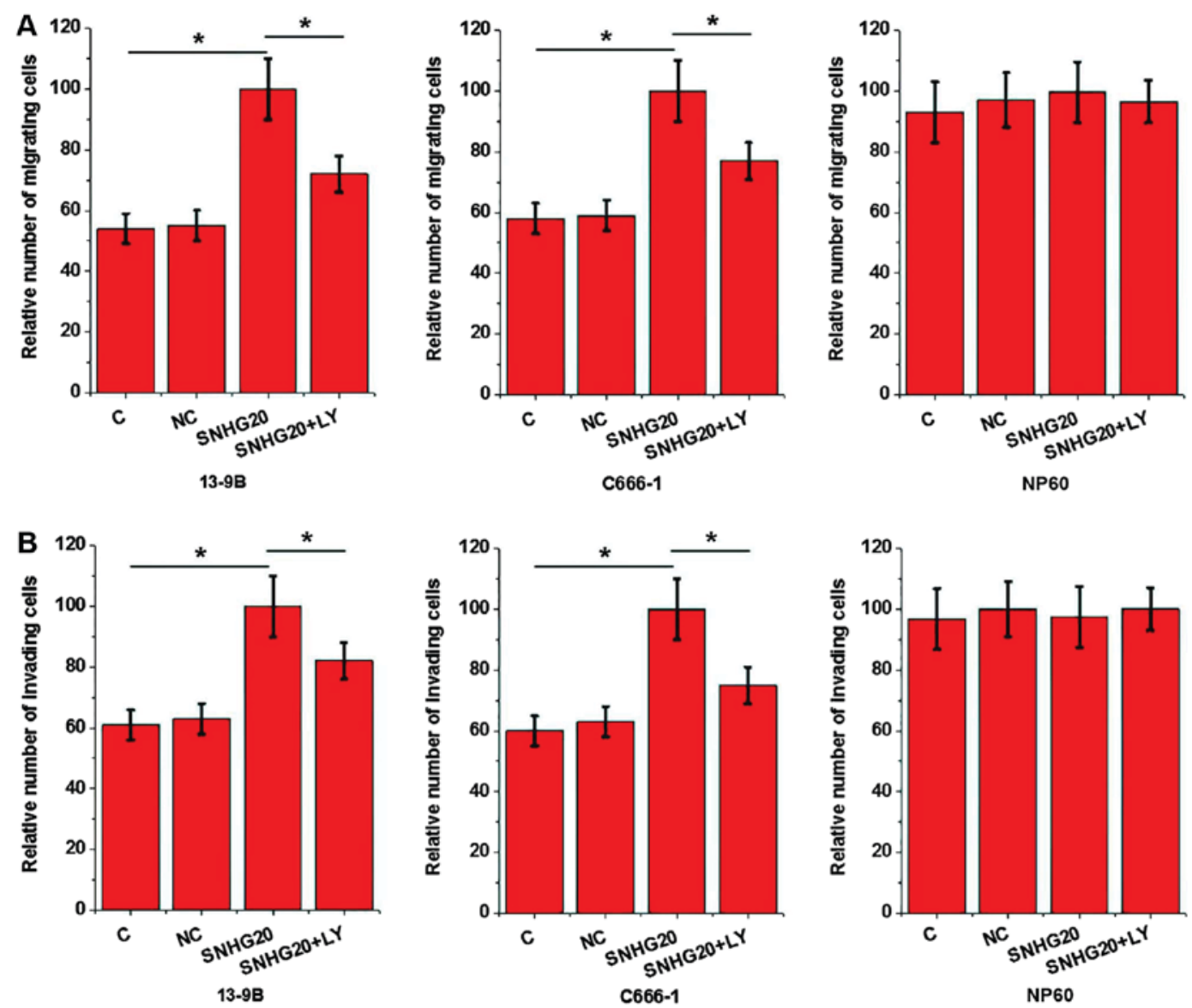

Figure 5. Effects of SNHG20 overexpression and treatment with TGF- $\beta$ inhibitor on cell migration and invasion. Alterations in (A) cell migration and (B) cell invasion. "P<0.05. LY, LY2109761; C, untransfected cells; NC, cells transfected with empty vectors; SNHG20, small nucleolar RNA host gene 20. 
SNHG20 in the diagnosis of nasopharyngeal carcinoma. In the present study, no significant associations were identified between serum levels of SNHG20 and patients' age, sex, and smoking and alcohol consumption habits. Therefore, serum SNHG20 may serve as a reliable biomarker for the diagnosis and prognosis of nasopharyngeal carcinoma. As previously demonstrated, SNHG20 expression is upregulated in different types of malignancies $(9,10)$. Therefore, those malignancies should be excluded and/or multiple biomarkers should be combined to improve the diagnosis of nasopharyngeal carcinoma.

The present study also indicated that serum expression levels of SNHG20 were significantly associated with distant tumor metastasis, indicating the possible roles of SNHG20 in tumor cell invasion and migration. Transwell migration and invasion assays in the present study confirmed that SNHG20 promoted migration and invasion of human nasopharyngeal carcinoma cell lines and not the normal human nasopharyngeal epithelial cell line NP69. The TGF- $\beta$ signaling pathway is a regulator of metastasis of various types of cancer $(18,19)$, including nasopharyngeal carcinoma (12). Inhibition of TGF- $\beta$ suppresses tumor metastasis $(20,21)$. In the present study, SNHG20 overexpression promoted TGF- $\beta 1$ expression in nasopharyngeal carcinoma cells, while treatment with TGF- $\beta 1$ exhibited no significant effects on the expression levels of SNHG20. Furthermore, treatment with TGF- $\beta$ inhibitor LY2109761 also significantly reduced the effects of SNHG20 overexpression on migration and invasion of nasopharyngeal carcinoma cells. In addition, in the present study, oncogenic signaling pathways including Wnt, Akt and $\mathrm{NF}-\kappa \mathrm{B}$ failed to respond after SNHG20 overexpression. The results of the present study suggested that SNHG20 may promote the metastasis of nasopharyngeal carcinoma by upregulating TGF- $\beta 1$, but not other oncogenes, such as $\mathrm{NF}-\kappa \mathrm{B}$.

In conclusion, SNHG20 was upregulated in nasopharyngeal carcinoma. Serum expression level of SNHG20 could serve as a potential diagnostic and prognostic marker for nasopharyngeal carcinoma. SNHG20 overexpression promoted nasopharyngeal carcinoma cell migration and invasion, and increased the expression of TGF- $\beta 1$. TGF- $\beta$ inhibitor reduced the effects of SNHG20 overexpression on nasopharyngeal carcinoma cell migration and invasion, and exhibited no significant effects on SNHG20 expression. Therefore, the present study concluded that IncRNA SNHG20 may promote the metastasis of nasopharyngeal carcinoma cells by upregulating TGF- $\beta 1$.

\section{Acknowledgements}

Not applicable.

\section{Funding}

No funding was received.

\section{Availability of data and materials}

The datasets used and/or analyzed during the present study are available from the corresponding author on reasonable request.

\section{Authors' contributions}

CS, YS and EZ analyzed and interpreted the experimental data. CS was a major contributor in writing the manuscript. EZ revised the manuscript. All authors read and approved the final manuscript.

\section{Ethics approval and consent to participate}

The study was approved by the Ethics Committee of Wei Hai Hospital Affiliated to Medical College of Qing Dao University (Shandong, China).

\section{Patient consent for publication}

Not applicable.

\section{Competing interests}

The authors declare that they have no competing interests.

\section{References}

1. Wei WI and Sham JS: Nasopharyngeal carcinoma. Lancet 365: 2041-2054, 2005

2. Du M, Chen W, Zhang W, Tian XK, Wang T, Wu J, Gu J, Zhang N, Lu ZW, Qian LX, et al: TGF-? regulates the ERK/MAPK pathway independent of the SMAD pathway by repressing miRNA-124 to increase MALAT1 expression in nasopharyngeal carcinoma. Biomed Pharmacother 99: 688-696, 2018.

3. Wang Y, Zhang Y and Ma S: Racial differences in nasopharyngeal carcinoma in the United States. Cancer Epidemiol 37: 793-802, 2013.

4. Lee AWM, Tung SY, Ng WT, Lee V, Ngan RKC, Choi HCW, Chan LLK, Siu LL, Ng AWY, Leung TW, et al: A multicenter, phase 3, randomized trial of concurrent chemoradiotherapy plus adjuvant chemotherapy versus radiotherapy alone in patients with regionally advanced nasopharyngeal carcinoma: 10-year outcomes for efficacy and toxicity. Cancer 123: 4147-4157, 2017.

5. Lee V, Kwong D, Leung TW, Lam KO, Tong CC and Lee A: Palliative systemic therapy for recurrent or metastatic nasopharyngeal carcinoma-How far have we achieved? Crit Rev Oncol Hematol 114: 13-23, 2017.

6. Chan AT: Nasopharyngeal carcinoma. Ann Oncol 21 (Suppl 7): vii308-vii312, 2010

7. Gao W, Chan JY and Wong TS: Differential expression of long noncoding RNA in primary and recurrent nasopharyngeal carcinoma. Biomed Res Int 2014: 404567, 2014.

8. Wang YN, Shan K, Yao MD, Yao J, Wang JJ, Li X, Liu B, Zhang YY,Ji Y, Jiang Q and Yan B: Long noncoding RNA-GAS5: A novel regulator of hypertension-induced vascular remodeling. Hypertension 68: 736-748, 2016.

9. Zhang D, Cao C, Liu L and Wu D: Up-regulation of LncRNA SNHG20 predicts poor prognosis in hepatocellular carcinoma. J Cancer 7: 608-617, 2016 .

10. Li C, Zhou L, He J, Fang XQ, Zhu SW and Xiong MM: Increased long noncoding RNA SNHG20 predicts poor prognosis in colorectal cancer. BMC Cancer 16: 655, 2016.

11. Livak KJ and Schmittgen TD: Analysis of relative gene expression data using real-time quantitative PCR and the 2(-Delta Delta C(T)) method. Methods 25: 402-408, 2001

12. Wu Y, Shen Z, Wang K, Ha Y, Lei H, Jia Y, Ding R, Wu D, Gan S, Li R, et al: High FMNL3 expression promotes nasopharyngeal carcinoma cell metastasis: Role in TGF- $\beta 1$-induced epithelia-to-mesenchymal transition. Sci Rep 7: 42507, 2017.

13. Schwarzenbach H, Hoon DS and Pantel K: Cell-free nucleic acids as biomarkers in cancer patients. Nat Rev Cancer 11: 426-437, 2011.

14. Cho WC: Nasopharyngeal carcinoma: Molecular biomarker discovery and progress. Mol Cancer 6: 1, 2007. 
15. Bianchessi V, Badi I, Bertolotti M, Nigro P, D'Alessandra Y, Capogrossi MC, Zanobini M, Pompilio G, Raucci A and Lauri A: The mitochondrial lncRNA ASncmtRNA-2 is induced in aging and replicative senescence in endothelial cells. $\mathrm{J} \mathrm{Mol}$ Cell Cardiol 81: 62-70, 2015.

16. Lu L, Xu H, Luo F, Liu X, Lu X, Yang Q, Xue J, Chen C, Shi L and Liu Q: Epigenetic silencing of miR-218 by the lncRNA CCAT1, acting via BMI1, promotes an altered cell cycle transition in the malignant transformation of HBE cells induced by cigarette smoke extract. Toxicol Appl Pharmacol 304: 30-41, 2016.

17. Kryger RL, Fan L, Jaquet V and Wilce PA: Identification of a ncRNA which is elevated in brain of human alcoholics and alcohol-treated rats. Alcohol Clin Exp Res 30: 152A, 2006.

18. Yin JJ, Selander K, Chirgwin JM, Dallas M, Grubbs BG, Wieser R, Massagué J, Mundy GR and Guise TA: TGF-beta signaling blockade inhibits PTHrP secretion by breast cancer cells and bone metastases development. J Clin Invest 103: 197-206, 1999.
19. Muraoka RS, Dumont N, Ritter CA, Dugger TC, Brantley DM, Chen J, Easterly E, Roebuck LR, Ryan S, Gotwals PJ, et al: Blockade of TGF-beta inhibits mammary tumor cell viability, migration, and metastases. J Clin Invest 109: 1551-1559, 2002.

20. Siegel PM and Massagué J: Cytostatic and apoptotic actions of TGF-beta in homeostasis and cancer. Nat Rev Cancer 3: 807-821, 2003.

21. Yang YA, Dukhanina O, Tang B, Mamura M, Letterio JJ, MacGregor J, Patel SC, Khozin S, Liu ZY, Green J, et al: Lifetime exposure to a soluble TGF- $\beta$ antagonist protects mice against metastasis without adverse side effects. JClin Invest 109: 1607-1615, 2002.

This work is licensed under a Creative Commons Attribution-NonCommercial-NoDerivatives 4.0 International (CC BY-NC-ND 4.0) License. 